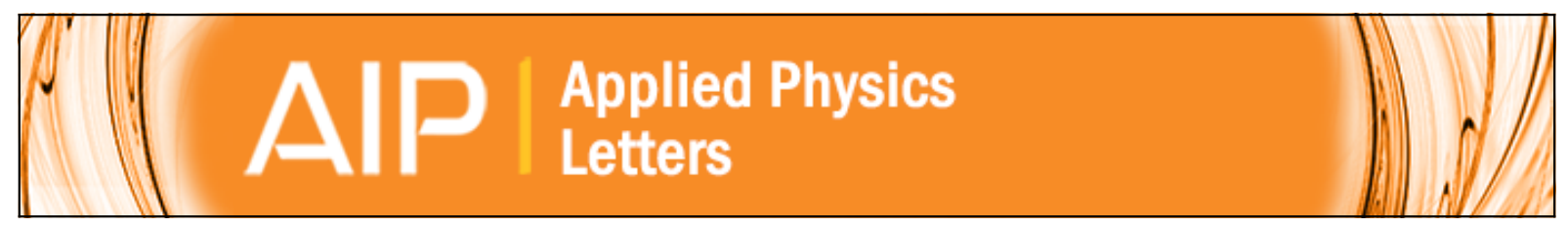

\title{
Formation of polybromine anions and concurrent heavy hole doping in carbon nanotubes
}

Dongchul Sung, Noejung Park, Wanjun Park, and Suklyun Hong

Citation: Applied Physics Letters 90, 093502 (2007); doi: 10.1063/1.2710471

View online: http://dx.doi.org/10.1063/1.2710471

View Table of Contents: http://scitation.aip.org/content/aip/journal/apl/90/9?ver=pdfcov

Published by the AIP Publishing

\section{Articles you may be interested in}

Preparing spin-polarized scanning tunneling microscope probes on capped carbon nanotubes by Fe doping: A first-principles study

Appl. Phys. Lett. 94, 193106 (2009); 10.1063/1.3134483

Changes of the electronic structure of a $(8,0)$ zigzag nanotube due to doping with potassium

Low Temp. Phys. 35, 137 (2009); 10.1063/1.3075944

Defect states in carbon nanotubes and related band structure engineering: A first-principles study

J. Appl. Phys. 105, 024312 (2009); 10.1063/1.3072695

Controllable $\mathrm{p}$ and $\mathrm{n}$ doping of single-walled carbon nanotubes by encapsulation of organic molecules and fullerene: A theoretical investigation

Appl. Phys. Lett. 86, 223113 (2005); 10.1063/1.1941476

Magnetism in BN nanotubes induced by carbon doping

Appl. Phys. Lett. 86, 122510 (2005); 10.1063/1.1890477

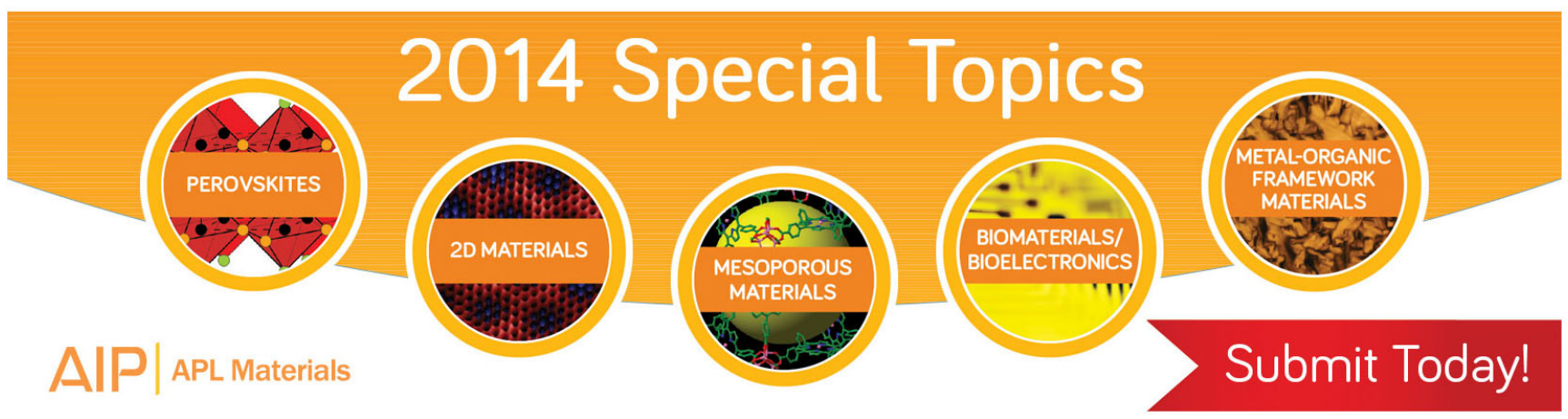




\title{
Formation of polybromine anions and concurrent heavy hole doping in carbon nanotubes
}

\author{
Dongchul Sung \\ Department of Physics and Institute of Fundamental Physics, Sejong University, Seoul 143-747, Korea and \\ Device Laboratory, Samsung Advanced Institute of Technology, Suwon 440-600, Korea \\ Noejung Park ${ }^{\mathrm{a}), \mathrm{b})}$ \\ Department of Applied Physics, Dankook University, Seoul 140-714, Korea
}

Wanjun Park

Department of Electronics and Computer Engineering, Hanyang University, Seoul 133-791, Korea

Suklyun Hong ${ }^{\mathrm{a}, \mathrm{c})}$

Department of Physics and Institute of Fundamental Physics, Sejong University, Seoul 143-747, Korea

(Received 18 September 2006; accepted 26 January 2007; published online 27 February 2007)

\begin{abstract}
Using density-functional theory calculations, we investigate the atomic and electronic structure of the bromine species encapsulated in carbon nanotubes. We find that the odd-membered molecular structures $\left(\mathrm{Br}_{3}\right.$ and $\left.\mathrm{Br}_{5}\right)$ are energetically favored than the common $\mathrm{Br}_{2}$ molecule. The transformation from bromine molecules $\left(\mathrm{Br}_{2}\right)$ into $\mathrm{Br}_{3}$ or $\mathrm{Br}_{5}$ is found to be almost barrierless. A strong electron transfer from the nanotube to the adsorbates, which has been doubtful in previous studies, is accompanied by the formation of such odd-membered polybromine anions. We suggest that the tip-opened carbon nanotube samples can be heavily hole-doped after exposure to $\mathrm{Br}_{2}$ gas. (C) 2007 American Institute of Physics. [DOI: 10.1063/1.2710471]
\end{abstract}

The fascinating material properties of the carbon nanotube $(\mathrm{CNT})$ have generated immense interest from researchers in various fields. In particular, its intrinsic onedimensional geometry and excellent transport properties make the nanotube one of the prime candidates for active elements in next generation electronic devices. ${ }^{1-3}$ Examples of the potential CNT applications include CNT-based switching devices, field emitters for flat-panel displays, memory or logic circuits, and sensors. ${ }^{4-8}$ Despite that no method to date has succeeded in the controlled growth of either metallic or semiconducting nanotubes, their selective use has been highly desirable for practical applications. In this regard, controlling the electronic properties of CNT has become a vital issue and thus needs fundamental understanding of its atomic and electronic structure.

Adsorptions of halogen elements on the nanotube have been tried to exploit its electronic structure. ${ }^{9,10}$ Especially, a sharp increase in the conductance was shown to accompany the intercalation of bromine molecules. ${ }^{11,12}$ Theoretical studies have investigated the adsorption energetics and electronic structure of the Br/CNT system. ${ }^{13,14}$ The calculations revealed a large amount of energy gain upon the encapsulation of $\mathrm{Br}_{2}$ inside the small-diameter CNT $\left[0.72 \mathrm{eV} / \mathrm{Br}_{2}\right.$ in the $(10,0) \mathrm{CNT}] .{ }^{13}$ On the surface of the large-diameter nanotube, the adsorption energy was found to be about $0.1 \mathrm{eV} / \mathrm{Br}$, whereas the metallic nanotube showed a slightly larger binding energy. ${ }^{13,14}$ However, these theoretical studies have led to an unexplained question about the reality and possibility of hole doping of the semiconducting nanotube with bromine molecular vapors. Note that the charge transfer

\footnotetext{
a) Authors to whom correspondence should be addressed.

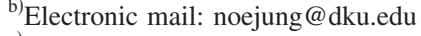

${ }^{c)}$ Electronic mail: hong@sejong.ac.kr
}

from the nanotube to the physisorbed $\mathrm{Br}_{2}$ molecule was calculated to be very small. ${ }^{14}$

In this letter we investigate the geometric and electronic structure of the bromine-encapsulated CNTs. We find that encapsulated bromine molecules $\left(\mathrm{Br}_{2}\right)$ transform into the charged polybromine species $\left(\mathrm{Br}_{3}^{-}\right.$and $\left.\mathrm{Br}_{5}^{-}\right)$, rendering the nanotube template heavily hole-doped. Thus the observed conductance increase upon the bromine doping in previous experiments ${ }^{11,12}$ is likely due to the formation of these polybromine anions. Throughout this work, total energy calculations are performed with the local-density approximation (LDA) and the generalized gradient-corrected approximation (GGA) in some limited cases, using the Vienna $a b$ initio simulation package (VASP). ${ }^{15}$ The ionic potentials are represented by the ultrasoft pseudopotentials as provided with VASP, ${ }^{16}$ and the energy cutoff for the plane-wave basis is set to $290 \mathrm{eV}$. The atomic positions are relaxed with residual forces smaller than $0.02 \mathrm{eV} / \AA$.

First, we investigate the molecular structure of the bromine adsorbates inside CNTs of various diameters [from $(10,0)$ to $(16,0)]$. Figure 1 shows the optimized geometries for $\mathrm{Br}_{2}, \mathrm{Br}_{3}, \mathrm{Br}_{4}$, and $\mathrm{Br}_{5}$ encapsulated inside the $(16,0)$ CNT. The unit cell dimensions along the axial direction of the nanotube are set to triple of the minimal unit cell of the zigzag nanotube $(12.735 \AA$ ) for Figs. 1(a) and 1(b) and to quadruple of that (16.98 $\AA$ ) for Figs. 1(c) and 1(d). We observe that $\mathrm{Br}_{2}$ and $\mathrm{Br}_{3}$ are aligned in the nanotube with the $\mathrm{Br}-\mathrm{Br}$ bond lengths of 2.361 and $2.532 \AA$, respectively, as shown in Figs. 1(a) and 1(b). The bond length $(2.361 \AA)$ is a little longer than the interatomic distance $\left(2.274 \AA\right.$ ) of $\mathrm{Br}_{2}$ in the vacuum. Two $\mathrm{Br}-\mathrm{Br}$ bonds $\left(2.575 \AA\right.$ ) at the edges of $\mathrm{Br}_{4}$ in Fig. 1(c) are mostly aligned with the CNT axis, while the central bond $(2.582 \AA)$ deviates a little. Noticeably, $\mathrm{Br}_{5}$ forms a bent molecule as shown in Fig. 1(d), where the bond lengths at the edges of $\mathrm{Br}_{5}$ are $2.452 \AA$, whereas those in the 

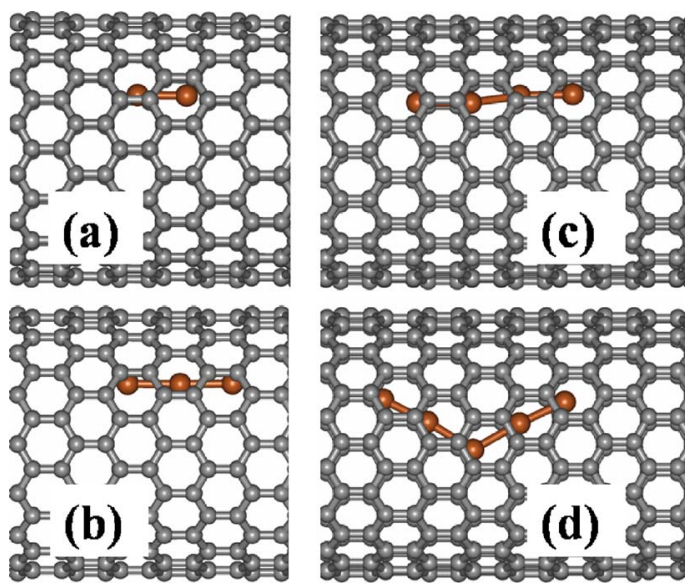

FIG. 1. (Color online) Optimized adsorption geometries of (a) $\mathrm{Br}_{2}$, (b) $\mathrm{Br}_{3}$, (c) $\mathrm{Br}_{4}$, and (d) $\mathrm{Br}_{5}$ inside the $(16,0)$ CNT.

center are $2.617 \AA$. It is noteworthy that the bromine adsorbates do not form chemical bonds with carbon atoms in the nanotube. The nearest $\mathrm{C}-\mathrm{Br}$ distances are 3.442, 3.397, 3.385, and $3.335 \AA$ for Figs. 1(a)-1(d), respectively. We also note that the molecular structures of $\mathrm{Br}_{3}, \mathrm{Br}_{4}$, and $\mathrm{Br}_{5}$, as shown in Fig. 1, are consistent with the previously calculated anionic polybromine clusters, in spite of the severe difference in the calculation scheme. ${ }^{17}$ Our results, together with the energetics shown below, indicate that such polyhalogen anions could stably exist inside the carbon nanotube. ${ }^{18}$

The binding strength for the bromine adsorbates inside nanotubes with various diameters is investigated mainly with LDA, as shown in Fig. 2. Here the formation energy is defined as $E=(1 / n)\left[E_{\text {tot }}\left(\mathrm{Br}_{n} / \mathrm{CNT}\right)-E_{\text {tot }}(\mathrm{CNT})\right.$ $\left.-(n / 2) E_{\text {tot }}\left(\mathrm{Br}_{2}\right)\right]$, where $n$ is the number of bromine atoms in the unit cell. The reference energies of $\mathrm{Br}_{2}$ and isolated CNT are calculated with the same calculation parameters. Indeed, we observe that the polyhalogen structures, $\mathrm{Br}_{3}$ and $\mathrm{Br}_{5}$, are much favored inside CNTs. The drops in the formation en-

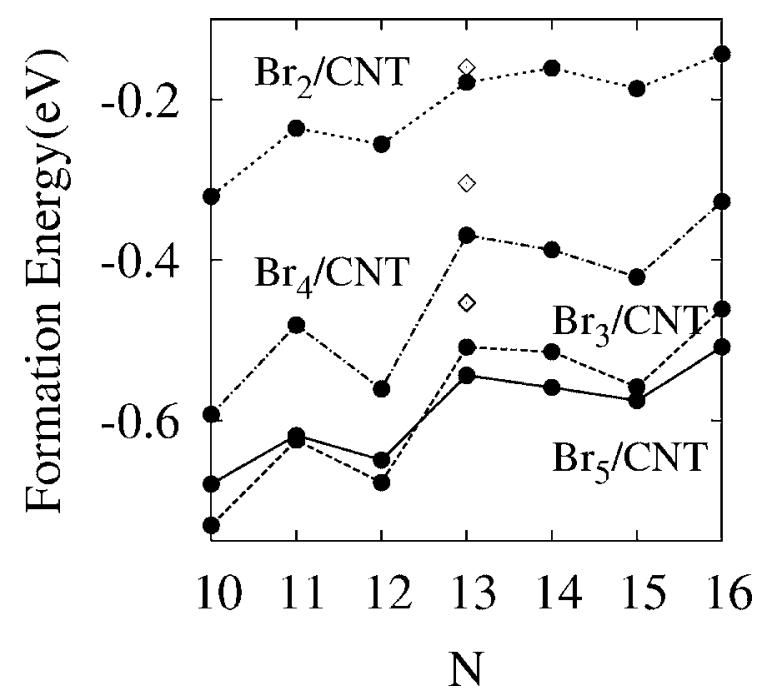

FIG. 2. Binding energies for bromine adsorbates encapsulated inside the $(N, 0)$ CNTs. Solid circles represent the data calculated with LDA. The dotted, dot-dashed, dashed, and solid lines connect the data for $\mathrm{Br}_{2}, \mathrm{Br}_{4}, \mathrm{Br}_{3}$, and $\mathrm{Br}_{5}$ encapsulated inside the nanotubes, respectively. The empty diamonds from top to bottom, calculated with GGA, correspond to the binding energy of $\mathrm{Br}_{2}, \mathrm{Br}_{4}, \mathrm{Br}_{3}$, and $\mathrm{Br}_{5}$ inside the $(13,0) \mathrm{CNT}$. The GGA data are downshifted by $0.1 \mathrm{eV}$ for visual convenience. Note that the GGA energy for $\mathrm{Br}_{3}$ inside the $(13,0) \mathrm{CNT}$ almost overlaps that of $\mathrm{Br}_{5}$.

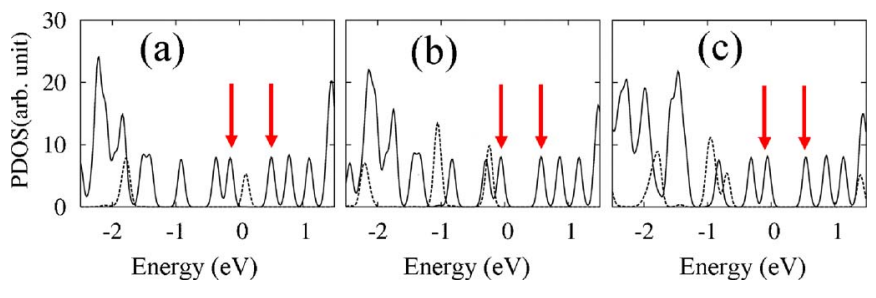

FIG. 3. (Color online) Projected densities of states for the nanotube (solid line) and bromine adsorbate (dotted lines) of the $(16,0) \mathrm{CNT}$, containing (a) $\mathrm{Br}_{2}$, (b) $\mathrm{Br}_{3}$, and (c) $\mathrm{Br}_{5}$, respectively. Two arrows indicate the valence and conduction band edges of CNT.

ergies at $N=12$ and $N=15$ imply that bromine adsorbates bind more strongly to the metallic nanotubes. We note that $\mathrm{Br}_{3}$ is more favored than $\mathrm{Br}_{5}$ in smaller nanotubes $\left(N=10,11\right.$, and 12), while $\mathrm{Br}_{5}$ is more favored in larger nanotubes. This should obviously be ascribed to the strain imposed on the $\mathrm{Br}_{5}$ molecule inside the small-diameter nanotubes. The linear chained $\mathrm{Br}_{4}$ is found to be more stable than $\mathrm{Br}_{2}$ but less stable than the odd-membered species inside the carbon nanotubes.

In these cases the bromine species are bound to the nanotubes by the weak intermolecular force in conjunction with ionic interactions. The weak intermolecular bindings, mediated by the van der Waals interaction, are estimated to be some halfway between the LDA and GGA results. ${ }^{19,20}$ In some cases, authors discussed that the LDA calculation effectively describes the weak intermolecular bindings while GGA greatly fails. ${ }^{21-23}$ For a cross-checking, we perform the GGA calculation only for the cases of $\mathrm{Br}_{2}, \mathrm{Br}_{3}, \mathrm{Br}_{4}$, and $\mathrm{Br}_{5}$ inside the $(13,0) \mathrm{CNT}$. We find much reduction of the binding strength in the GGA results, as shown in Fig. 2. This reduction should be ascribed to the underestimation of GGA for the weak intermolecular interaction between $\mathrm{Br}$ species and CNT. Nevertheless, we observe that the $\mathrm{Br}_{4}, \mathrm{Br}_{3}$, and $\mathrm{Br}_{5}$ structures are still much stabilized than $\mathrm{Br}_{2}$ inside the $(13,0)$ CNT. A proper description of the van der Waals interaction may be similar to the LDA results or some halfway between the LDA and GGA results. However, the energetics regarding the formation of the odd-membered bromine species is believed to be correct, irrespective of density functionals.

We now investigate the electronic structure of the bromine-encapsulated nanotubes to account for the energetics discussed in the previous paragraphs. The projected densities of states (PDOSs) for the CNT and adsorbates are presented with solid and dotted lines, respectively, in Fig. 3. Despite that bromine has been commonly considered as more electronegative than CNT, the lowest unoccupied molecular orbital level of $\mathrm{Br}_{2}$ is found to be in the midgap of the semiconducting CNT, as shown in Fig. 3(a). This means that the electron transfer from the nanotube to the $\mathrm{Br}_{2}$ should not be substantial, consistent with the usual physisorption feature. However, in the cases of $\mathrm{Br}_{3}$ and $\mathrm{Br}_{5}$ in $(16,0) \mathrm{CNT}$, the singly occupied molecular orbital (SOMO) levels are well below the Fermi level, rendering the valence band of the nanotube to be crossed by the Fermi level. Such features shown in Figs. 3(b) and 3(c) directly indicate an almost one electron transfer from the nanotube to the adsorbates $\left(\mathrm{Br}_{3}\right.$ or $\left.\mathrm{Br}_{5}\right)$.

For reference, the total energies of the isolated chargeneutral $\mathrm{Br}_{2}, \mathrm{Br}_{3}$, and $\mathrm{Br}_{5}$ structures are calculated with the same density-functional parameters. While $\mathrm{Br}_{3}$ is energetically unfavorable compared to $\mathrm{Br}_{2}$, the energy of the $\mathrm{Br}_{5}$ 
structure is found to be lower than that of $\mathrm{Br}_{2}$, which is largely consistent with the previous discussion: ${ }^{17}$ Total energies per atom are 0.0, 0.08, and $-0.09 \mathrm{eV}$ for $\mathrm{Br}_{2}, \mathrm{Br}_{3}$, and $\mathrm{Br}_{5}$, respectively, with respect to that of $\mathrm{Br}_{2}$ molecules. These energy values could directly be compared with those shown in Fig. 2. Note that there are partially occupied states, so-called SOMO states, at the Fermi level for the isolated $\mathrm{Br}_{3}$ and $\mathrm{Br}_{5}$ molecules, which become fully occupied when $\mathrm{Br}_{3}$ and $\mathrm{Br}_{5}$ are inside the nanotube. As noted above, these bromine species do not form chemical bonds with nanotube, and thus the nanotube band structures are almost rigid without disturbing its $\pi$ electronic structures, upon the electron transfer to the bromine species.

Finally, we investigate the energy barriers in the formation of these odd-membered bromine species. The optimized geometries of three $\mathrm{Br}_{2}$ molecules and two $\mathrm{Br}_{3}$ molecules in a row, encapsulated inside the $(16,0) \mathrm{CNT}$, are obtained in a long supercell $(25.44 \AA)$. Then, the energetics of the transformation from such three $\mathrm{Br}_{2}$ molecules into two $\mathrm{Br}_{3}$ structures is calculated with the nudged elastic band method. ${ }^{24}$ Initially, three far-separated $(6.05 \AA) \mathrm{Br}_{2}$ molecules approach with a very small energy barrier $(\approx 0.02 \mathrm{eV})$, which can be easily overcome at room temperature. They transform into the state of a weakly bonded two $\mathrm{Br}_{3}$ molecules and finally reach the well-separated two- $\mathrm{Br}_{3}$ state with a small energy barrier $(\approx 0.04 \mathrm{eV})$. In addition, to estimate the energy barrier in the formation of the $\mathrm{Br}_{5}$ structure, the $\mathrm{Br}_{3}$ and $\mathrm{Br}_{2}$ molecules are separated by the van der Waals distance in a row in the vacuum. The geometry optimization results in the bent $\mathrm{Br}_{5}$ structure spontaneously. Thus, provided that such $\mathrm{Br}_{2}$ and $\mathrm{Br}_{3}$ molecules exist in close vicinity inside the nanotube, their transformation barrier into the $\mathrm{Br}_{5}$ structure could also be negligible if the CNT diameter is large enough. Since the encapsulation of $\mathrm{Br}_{2}$ molecules is an exothermic process, as shown in Fig. 2, and since the transformation into the $\mathrm{Br}_{3}$ or $\mathrm{Br}_{5}$ species is almost barrierless, an exposure of tip-opened nanotube samples to a bromine partial pressure could result in the formation of charged $\mathrm{Br}_{3}$ or $\mathrm{Br}_{5}$ structures.

In summary, we investigated the atomic and electronic structure of the bromine-encapsulated CNT. It was found that the odd-membered molecular structures $\left(\mathrm{Br}_{3}\right.$ and $\left.\mathrm{Br}_{5}\right)$ are energetically favored inside CNTs rather than the common $\mathrm{Br}_{2}$ molecules. Their transformation of the $\mathrm{Br}_{2}$ molecules into the odd-membered structures is almost barrierless. The PDOS analysis reveals that the electron transfer becomes significant as the odd-membered species form inside CNT. We suggest that an exposure of tip-opened nanotubes to a modest bromine pressure could lead to functionalized, hole-doped nanotubes with an almost undamaged $\pi$ electronic structure.
This work was supported by Korea Research Foundation Grant funded by Korea Government (MOEHRD, Basic Research Promotion Fund) (KRF-2005-070-C00054), under which the calculations were performed by using the supercomputing resources of the Korea Institute of Science and Technology Information (KISTI). The research was also supported by grants from KOSEF through the Center for Nanotubes and Nanostructured Composites (CNNC). One of the authors (N.P.) would like to acknowledge the support from KISTI under the 7th Strategic Supercomputing Applications Support Program. Two of the authors (N.P. and W.P.) acknowledge the support from the Terra-level Nano Devices, Korea National Research Program.

${ }^{1}$ S. Frank, P. Poncharal, Z. L. Wang, and W. A. de Heer, Science 280, 1744 (1998).

${ }^{2}$ C. T. White and T. N. Todorov, Nature (London) 393, 240 (1998).

${ }^{3}$ W. Liang, M. Bockrath, D. Bozovic, J. H. Hafner, M. Tinkham, and H. Park, Nature (London) 411, 665 (2001).

${ }^{4}$ X. Zhou, J.-Y. Park, S. Huang, J. Liu, and P. L. McEuen, Phys. Rev. Lett. 95, 146805 (2005).

${ }^{5}$ T. Rueckes, K. Kim, E. Joselevich, G. Y. Tseng, C.-L. Cheung, and C. M. Lieber, Science 289, 94 (2000).

${ }^{6}$ A. Bachtold, P. Hadley, T. Nakanishi, and C. Dekker, Science 294, 1317 (2001).

${ }^{7}$ J. Kong, N. R. Franklin, C. Zhou, M. G. Chapline, S. Peng, K. Cho, and H. Dai, Science 287, 622 (2000).

${ }^{8}$ K. Besteman, J.-O. Lee, F. G. M. Wiertz, H. A. Heering, and C. Dekker, Nano Lett. 3, 727 (2003).

${ }^{9}$ X. Fan, E. C. Dickey, P. C. Eklund, K. A. Williams, L. Grigorian, R. Buczko, S. T. Pantelides, and S. J. Pennycook, Phys. Rev. Lett. 84, 4621 (2000).

${ }^{10}$ L. Grigorian, K. A. Williams, S. Fang, G. U. Sumanasekera, A. L. Loper, E. C. Dickey, S. J. Pennycook, and P. C. Eklund, Phys. Rev. Lett. 80, 5560 (1998).

${ }^{11}$ A. M. Rao, P. C. Eklund, S. Bandow, A. Thess, and R. E. Smalley, Nature (London) 388, 257 (1997).

${ }^{12}$ R. S. Lee, H. J. Kim, J. E. Fischer, A. Thess, and R. E. Smalley, Nature (London) 388, 255 (1997).

${ }^{13}$ N. Park, Y. Miyamoto, K. Lee, W. Choi, J. Ihm, J. Yu, and S. Han, Chem. Phys. Lett. 403, 135 (2005).

${ }^{14}$ S.-H. Jhi, S. G. Louie, and M. L. Cohen, Solid State Commun. 123, 495 (2002).

${ }^{15}$ G. Kresse and J. Furthmüller, Phys. Rev. B 54, 11169 (1996); Comput. Mater. Sci. 6, 15 (1996).

${ }^{16}$ G. Kresse and J. Hafner, J. Phys.: Condens. Matter 6, 8245 (1994).

${ }^{17}$ P. Schuster, H. Mikosch, and G. Bauer, J. Chem. Phys. 109, 1833 (1998).

${ }^{18}$ K. E. Nizzi, C. A. Pommerening, and L. S. Sunderlin, J. Phys. Chem. A 102, 7674 (1998).

${ }^{19}$ F. Ortmann, W. G. Schmidt, and F. Bechstedt, Phys. Rev. Lett. 95, 186101 (2005).

${ }^{20}$ Y.-H. Kim, Y. Zhao, A. Williamson, M. J. Heben, and S. B. Zhang, Phys. Rev. Lett. 96, 016102 (2006).

${ }^{21}$ Y. Okamoto and Y. Miyamoto, J. Phys. Chem. 105, 3470 (2001).

${ }^{22}$ M. Hasegawa and K. Nishidate, Phys. Rev. B 70, 205431 (2004).

${ }^{23}$ F. Tournus, J.-C. Charlier, and P. Mélinon, J. Chem. Phys. 122, 094315 (2005).

${ }^{24}$ G. Henkelman and H. Jónsson, J. Chem. Phys. 113, 9978 (2000); G. Henkelman, B. P. Uberuaga, and H. Jónsson, ibid. 113, 9901 (2000). 